\title{
Sentiment Analysis of Twitter Data (saotd)
}

\section{Evan L. Munson ${ }^{1}$, Christopher M. Smith ${ }^{1}$, Bradley C. Boehmke ${ }^{1}$, and Jason K. Freels ${ }^{1}$}

1 Air Force Institure of Technology

DOI: $10.21105 /$ joss.00764

\section{Software}

- Review 七

- Repository ca

- Archive ${ }^{\pi}$

Submitted: 17 April 2018

Published: 27 February 2019

\section{License}

Authors of papers retain copyright and release the work under a Creative Commons Attribution 4.0 International License (CC-BY). saotd is an $\mathrm{R}$ package that provides a programmatic interface to the Twitter API and can be used to acquire tweets based on user-specified \#hashtags. The package will clean and tidy the Twitter data, determine the latent topics within the tweets utilizing Latent Dirichlet Allocation (LDA), determine a sentiment score using the Bing lexicon dictionary, and create output visualizations.

The package is available on GitHub and archived on Zenodo. To configure the package a user must follow these steps:

- Creating a personal Twitter account if they don't already have one.

- Enable a Twitter Developers Account.

- Create an application which will provide them with API access tokens required by the saotd package to function.

The package is laid out in five different categories: Acquire, Explore, Topic Analysis, Sentiment Calculation, and Visualizations.

- Acquire allows a user to acquire tweets of their choosing by accessing the Twitter API.

- Explore provides functions to tidy, explore unigrams, bigrams, tri-grams, in addition to bigram netwrorks and correlation networks.

- Topic analysis allows a user to explore the latent topics buried within the tweets.

- Sentiment calculation utilizes the Bing lexicon dictionary to score the text ( $\mathrm{Hu} \&$ Liu, 2004).

- Visualizations allow the user to better understand the sentiment of the tweets.

The package utilizes tidy dataframes and therefore depends on the tidyverse package (Wickham, 2017) and additionally uses the tidytext package (Silge \& Robinson, 2017). The number of latent topics is determined using the ldatuning package (Nikita, 2016) and the latent dirichlet allocation (LDA) topics is determined using the topicmodels package (Grün \& Hornik, 2011).

The saotd package has research applications in many disciplines which need to access tweets from the Twitter platform and carry out sentiment analyses. This package was created to quickly determine the sentiment of Twitter and to inform analysts on the opinions contained within tweets.

\section{References}

Grün, B., \& Hornik, K. (2011). topicmodels: An R package for fitting topic models. Journal of Statistical Software, 40(13), 1-30. doi:10.18637/jss.v040.i13 
Hu, M., \& Liu, B. (2004). Mining and summarizing customer reviews. In Proceedings of the tenth acm sigkdd international conference on knowledge discovery and data mining (pp. 168-177). ACM.

Nikita, M. (2016). Ldatuning: Tuning of the latent dirichlet allocation models parameters. Retrieved from https://CRAN.R-project.org/package=ldatuning

Silge, J., \& Robinson, D. (2017). Text Mining with R a Tidy Approach. (N. Tache, Ed.) (1st ed., p. 178). Sebastopol: O'Reilly. Retrieved from http://tidytextmining.com/

Wickham, H. (2017). Tidyverse: Easily install and load the 'tidyverse'. Retrieved from https://CRAN.R-project.org/package=tidyverse 